\title{
THE ROLE OF ANTHROPOMETRY IN ACUTE ST-ELEVATION MYOCARDIAL INFARCTION TREATED WITH PRIMARY PERCUTANEOUS CORONARY INTERVENTION
}

\author{
Marko Mornar Jelavić ${ }^{1}$ Zdravko Babić ${ }^{2}$, Hrvoje Pintarić3 ${ }^{3}$ and Marjeta Mišigoj-Duraković ${ }^{4}$ \\ ${ }^{1}$ Department of Internal Medicine and Dialysis, Zagreb-East Health Center; ${ }^{2}$ Coronary Care Unit, \\ ${ }^{3}$ Cardiac Catheterization Laboratory, Sestre milosrdnice University Hospital Center; \\ ${ }^{4}$ Department of Kinesiologic Anthropology, Faculty of Kinesiology, Zagreb, Croatia
}

\begin{abstract}
SUMMARY - The aim of this study was to investigate the controversial influence of anthropometry on clinical severity and prognosis of acute ST-elevation myocardial infarction (STEMI). We prospectively analyzed 250 patients with acute STEMI treated with primary percutaneous coronary intervention (September 2011 - September 2012). They were grouped according to the following anthropometric parameters: body mass index (BMI) $\left(<25.0,25.0-29.9, \geq 30.0 \mathrm{~kg} / \mathrm{m}^{2}\right)$, waist circumference (WC) $(<102 / 88, \geq 102 / 88 \mathrm{~cm})$, waist-to-hip ratio (WHR) $(<0.90 / 0.85, \geq 0.90 / 0.85)$ and waistto-height ratio (WHtR) $(<53 / 49,53 / 49-62 / 57, \geq 63 / 58)$. The groups were analyzed by baseline, as well as severity (clinical, laboratory, echocardiography, coronary angiography, in-hospital complications) and prognostic parameters (major adverse cardiovascular events and sick leave duration during 12-month follow up). Patients with BMI $<25.0 \mathrm{~kg} / \mathrm{m}^{2}$ had the highest rates of dyspnea and those with $\mathrm{BMI} \geq 30.0 \mathrm{~kg} / \mathrm{m}^{2}$ had the longest hospitalization and widest stents; patients with WHR $\geq 0.90 / 0.85$ had higher rates of significantly stenosed proximal/middle coronary segments, while those with $\mathrm{WHtR} \geq 63 / 58$ had the highest rates of heart failure and total in-hospital complications $(\mathrm{p}<0.05)$. BMI $<25.0 \mathrm{~kg} / \mathrm{m}^{2}$ increased (odds ratio (OR) 2.00, confidence interval (CI) [1.09-3.68], $\mathrm{p}=0.026$ ) and BMI 25.0-29.9 $\mathrm{kg} / \mathrm{m}^{2}$ reduced (OR 0.52, CI [0.30-0.91], $\mathrm{p}=0.022$ ) the risk of dyspnea; WHR $\geq 0.90 / 0.85$ increased the risk of significant proximal/middle coronary segment stenosis (OR 3.34, CI [1.13-9.86], $\mathrm{p}=0.029)$ and $\mathrm{WHtR} \geq 63 / 58$ the risk of heart failure (OR 2.05, CI [1.13-3.71], $\mathrm{p}=0.017$ ) and total in-hospital complications (OR 1.94, CI [1.13-3.33], $\mathrm{p}=0.017)(\mathrm{p}<0.05)$. In conclusion, WHR and WHtR are better anthropometric parameters than BMI in predicting acute STEMI severity, while WC has no influence on it. Anthropometry has no influence on prognosis.
\end{abstract}

Key words: Anthropometry; Obesity; Myocardial infarction; Percutaneous coronary intervention; Sick leave

\section{Introduction}

Overweight and obesity are one of the major public health problems. Worldwide, at least 1.4 billion adults are overweight, i.e. over 200 million men and nearly

Correspondence to: Marko Mornar Jelavic, MD, PhD, Department of Internal Medicine and Dialysis, Zagreb-East Health Center, Ninska 10, HR-10000 Zagreb, Croatia

E-mail: mjelavic@yahoo.com

Received November 2, 2015, accepted March 21, 2016
300 million women are obese ${ }^{1}$. Obesity prevalence in Europe has reached epidemic proportions (up to $28.3 \%$ of men and $36.5 \%$ of women) with higher prevalence rates in Central, Eastern, and Southern Europe than those in Western and Northern Europe ${ }^{2}$. Obesity is an independent risk factor for the development of cardiovascular disease (CVD), including coronary artery disease $(\mathrm{CAD})$ and heart failure. It is frequently associated with other CVD risk factors such as arterial hypertension, diabetes mellitus type 2, atherogenic 
dyslipidemia, as well as with an increased risk of allcause morbidity and mortality ${ }^{3}$. Measurement of body mass index (BMI), waist circumference (WC), waistto-hip ratio (WHR) and waist-to-height ratio (WHtR) is a primary method for diagnosing obesity. While BMI determines overall obesity, other anthropometric parameters are related to central obesity where body fat is primarily located in the abdomen. Central obesity measures, especially WHtR, are stronger predictors of CVD risk than $\mathrm{BMI}^{4,5}$. Central obesity correlates with excessive visceral fat, which is directly associated with insulin resistance and compensatory hyperinsulinemia, dyslipidemia and inflammatory states that synergistically lead to smooth muscle cell proliferation, calcium and cholesterol ester deposition in the artery, and finally to atherosclerotic vascular disease $^{6}$. In subjects with acute myocardial infarction (MI), there is positive association of abdominal obesity and decreasing BMI with higher mortality. This has been called the 'obesity paradox'. The possible explanation could be that BMI does not adequately discriminate the difference between body fat (especially abdominal) and lean muscle mass ${ }^{4}$.

Using the anthropometric parameters, the aims of this study were:

1. to evaluate the baseline data of patients with obesity and acute ST-elevation myocardial infarction (STEMI), and to compare the results with STEMI patients without obesity;

2. to evaluate the severity and prognosis of acute STEMI in patients with obesity, and to compare the results with STEMI patients without obesity; and

3. to investigate the controversial influence of anthropometric parameters on clinical severity and prognosis of acute STEMI, as well as their unknown influence on the involvement of coronary artery segments with significant stenosis and sick leave duration (SLD).

\section{Patients and Methods}

\section{Patient population}

This prospective monocentric study was performed on 250 consecutive patients with acute STEMI. They were treated with primary percutaneous coronary intervention (PCI) at Department of Cardiology, Sestre milosrdnice University Hospital Center (September
2011 - September 2012). The diagnosis was established and primary PCI performed using the criteria of the European Society of Cardiology $y^{7,8}$. The inclusion criteria were presenting within $12 \mathrm{~h}$ from the onset of symptoms (history of chest pain/discomfort lasting for 10-20 minutes or more, not responding fully to nitroglycerine), persistent ST-segment elevation on electrocardiography in at least two consecutive leads, or (presumed) new left bundle-branch block, and elevated cardiac laboratory biomarkers (cardiac troponin $\mathrm{T}(\mathrm{c} T \mathrm{n} \mathrm{T})$ and creatine kinase $(\mathrm{CK})$ ). The study was approved by the Ethics Committee of the Sestre milosrdnice University Hospital Center, Zagreb, Croatia.

\section{Data collection}

After primary PCI, patients were grouped according to anthropometric parameters as follows: BMI $\left(<25.0,25.0-29.9\right.$ and $\geq 30.0 \mathrm{~kg} / \mathrm{m}^{2}$ for normal weight, overweight and overall obesity, respectively), WC ( $<102 / 88$ and $\geq 102 / 88 \mathrm{~cm}$ for normal weight and central obesity in males/females, respectively), WHR $(<0.90 / 0.85$ and $\geq 0.90 / 0.85$ for normal weight and central obesity in males/females, respectively) and WHtR $(<53 / 49,53 / 49-62 / 57$ and $\geq 63 / 58$ for normal weight, overweight and central obesity in males/females, respectively) $)^{4,9}$. The groups were analyzed by baseline, as well as severity and prognostic parameters of acute STEMI.

\section{Baseline parameters}

Baseline demographic and medical history parameters included gender, age, arterial hypertension, dyslipidemia (elevated triglycerides and/or low HDLcholesterol), hyperglycemia, smoking status, known family history of cardiovascular events (MI, cerebrovascular insult), previous MI, previous PCI and coronary artery bypass grafting (CABG).

For the diagnosis of dyslipidemia, hypertension and hyperglycemia, we used the criteria published by the National Cholesterol Education Program - Adult Treatment Panel III group ${ }^{9}$, as follows:

1. hypertriglyceridemia: triglycerides $\geq 150 \mathrm{mg} / \mathrm{dL}$ $(1.7 \mathrm{mmol} / \mathrm{L})$, or on medication for elevated triglycerides;

2. low HDL-cholesterol: $<40 \mathrm{mg} / \mathrm{dL}(1.04 \mathrm{mmol} /$ $\mathrm{L})$ in males or $<50 \mathrm{mg} / \mathrm{dL}(1.29 \mathrm{mmol} / \mathrm{L})$ in 
females, or on medication for low HDL-cholesterol;

3. hypertension: blood pressure $\geq 130 / 85 \mathrm{~mm} \mathrm{Hg}$, or on medication for hypertension; and

4. hyperglycemia: fasting plasma glucose $\geq 100$ $\mathrm{mg} / \mathrm{dL}(5.6 \mathrm{mmol} / \mathrm{L})$, or on medication for hyperglycemia.

\section{Severity parameters}

The severity of acute STEMI was estimated by clinical presentation (angina pectoris, dyspnea, and length of hospitalization), in-hospital complications (arrhythmias, conduction disturbances, reperfusion arrhythmias, heart failure, cardiogenic shock, cardiac arrest, mechanical ventilation, reinfarction, repeated PCI, mortality, and total in-hospital complications), laboratory (maximal cTnT, CK), echocardiography (left ventricular ejection fraction, $\mathrm{LVEF}$ ) and coronary angiography findings.

Serum CK activity was measured by spectrophotometry (Olympus 680, Beckman Coulter Inc., California, USA). Serum cTnT levels were measured by electrochemiluminescence (ECL) assay (Cobas e411, Roche Diagnostics, Sussex, UK). During hospital stay, echocardiography (Acuson Sequoia 512, Siemens, Munich, Germany) was performed in all patients according to the clinical standards and current echocardiography guidelines ${ }^{10}$.

Coronary angiography was performed by applying a monoplane system (Axiom Artis, Siemens, Erlangen, Germany) by a common technique, as recommended in current guidelines ${ }^{8}$. Patients received $70 \mathrm{IE} / \mathrm{kg}$ unfractionated heparin, $300 \mathrm{mg}$ aspirin, a loading dose of $600 \mathrm{mg}$ clopidogrel, and a GPIIb/IIIa inhibitor according to judgment of interventional cardiologist. Coronary artery stenosis of more than 50\% was considered clinically significant. We analyzed the number of significantly narrowed coronary arteries, and number, length and diameter of stents used. Additionally, for the first time, we analyzed significantly stenosed segments of coronary arteries. For that purpose, and according to the modified American Heart Association classification ${ }^{11}$, coronary arteries were divided into 16 segments. Segments were classified in two groups, as follows:

1. proximal and middle coronary segments: segment 1 (right coronary artery (RCA), proximal), segment 2 (RCA, mid), segment 5 (main stem), segment 6 (left anterior descending coronary artery (LAD), proximal), segment 7 (LAD, mid), segment 9 (first diagonal), segment 11 (left circumflex artery (LCX), proximal), segment 12 (obtuse marginal); and

2. distal coronary segments: segment 3 (RCA, distal), segment 4 (right posterior descendens), segment 8 (LAD, distal), segment 10 (second diagonal), segment 13 (LCX, distal), segment 14 (LCX, posterolateral branch), segment 15 (LCX, posterodescendens branch), segment 16 (RCA, posterolateral branch).

\section{Prognostic parameters}

The prognosis of acute STEMI was estimated using major adverse cardiovascular events (MACE) parameters (reinfarction, coronary artery restenosis and/ or new stenosis, cardiac and non-cardiac rehospitalization, cerebrovascular insult, urgent $\mathrm{CABG}$, mortality, total MACE) during 12-month follow up. Data were collected by medical examination, checking medical documentation, or telephone contact with patients, family members or home physicians. In addition, during the same follow up period, we collected data on SLD of working population.

\section{Statistical analysis}

Qualitative data were expressed as absolute number and percentage. We used $\chi^{2}$-test with Yates correction for comparison and analysis. Quantitative data were expressed as median and corresponding interquartile range. Differences between two groups were tested by Mann-Whitney U test. Differences among three groups were tested by nonparametric analysis of variance (Kruskal-Wallis ANOVA). Logistic regression analysis was used to investigate the relationship between one dependent and one or more independent variables that may influence or predict the value of the dependent variable. The limit of statistical significance was set at $\mathrm{p}<0.05$. Processing was done using the STATISTICA 6.0 for Windows software.

\section{Results}

Among 250 patients, there were $72(28.8 \%)$ patients with BMI $\geq 30.0 \mathrm{~kg} / \mathrm{m}^{2}, 149$ (59.6\%) with WC $\geq 102 / 88 \mathrm{~cm}, 222$ (88.8\%) with WHR $\geq 0.90 / 0.85$ and 
Table 1. Baseline characteristics of patients according to $B M I\left(\mathrm{~kg} / \mathrm{m}^{2}\right)$ and $W C(\mathrm{~cm})$

\begin{tabular}{|l|c|c|c|c|c|c|c|}
\hline Parameter & $\begin{array}{c}\mathrm{BMI}<25.0 \\
(\mathrm{n}=60)\end{array}$ & $\begin{array}{c}\text { BMI } \\
25.0-29.9 \\
(\mathrm{n}=118)\end{array}$ & $\begin{array}{c}\mathrm{BMI} \geq 30.0 \\
(\mathrm{n}=72)\end{array}$ & $\mathrm{p}$ & $\begin{array}{c}\text { WC } \\
<02 / 88 \\
(\mathrm{n}=101)\end{array}$ & $\begin{array}{c}\text { WC } \\
\geq 102 / 88 \\
(\mathrm{n}=149)\end{array}$ & $\mathrm{p}$ \\
\hline Men, n (\%) & $37(61.7)$ & $88(74.6)$ & $52(72.2)$ & 0.191 & $87(86.1)$ & $90(60.4)$ & $\mathbf{0 . 0 0 0}$ \\
Women, n (\%) & $23(38.3)$ & $30(25.4)$ & $20(27.8)$ & 0.191 & $14(13.9)$ & $59(39.6)$ & $\mathbf{0 . 0 0 0}$ \\
Age (years) & $64(40-90)$ & $62(25-92)$ & $61(39-85)$ & 0.201 & $60(41-90)$ & $63(25-92)$ & 0.080 \\
Arterial hypertension, n (\%) & $34(56.7)$ & $84(71.2)$ & $63(87.5)$ & $\mathbf{0 . 0 0 0}$ & $54(53.5)$ & $127(85.2)$ & $\mathbf{0 . 0 0 0}$ \\
Dyslipidemia, n (\%) & $40(66.7)$ & $88(74.6)$ & $62(86.1)$ & $\mathbf{0 . 0 3 0}$ & $72(71.3)$ & $118(79.2)$ & 0.151 \\
Hyperglycemia, n (\%) & $13(21.7)$ & $26(22)$ & $22(30.6)$ & 0.353 & $23(22.8)$ & $38(25.5)$ & 0.622 \\
Smoking, n (\%) & $37(61.7)$ & $54(45.8)$ & $38(52.8)$ & 0.130 & $57(56.4)$ & $72(48.3)$ & 0.208 \\
Family history, n (\%) & $19(31.7)$ & $55(46.6)$ & $33(45.8)$ & 0.135 & $44(43.6)$ & $63(42.3)$ & 0.841 \\
Previous MI, n (\%) & $5(8.3)$ & $10(8.5)$ & $7(9.7)$ & 0.948 & $10(9.9)$ & $12(8.1)$ & 0.613 \\
Previous PCI, n (\%) & $5(8.3)$ & $11(9.3)$ & $7(9.7)$ & 0.961 & $11(10.9)$ & $12(8.1)$ & 0.442 \\
Previous CABG, n (\%) & $0(0)$ & $0(0)$ & $1(1.4)$ & - & $0(0)$ & $1(0.7)$ & - \\
\hline
\end{tabular}

$\mathrm{BMI}=$ body mass index; $\mathrm{CABG}=$ coronary artery bypass graft $\mathrm{MI}=$ myocardial infarction; $\mathrm{PCI}=$ percutaneous coronary intervention; $\mathrm{WC}=$ waist circumference

81 (32.4\%) with WHtR $\geq 63 / 58$. We recorded the following results:

1) BMIs $\geq 30.0 \mathrm{~kg} / \mathrm{m}^{2}$ had the highest rates of hypertension and dyslipidemia, the longest hospitalization and widest stents, while BMIs $<25.0 \mathrm{~kg} / \mathrm{m}^{2}$ had the highest rates of dyspnea $(\mathrm{p}<0.05)$. Other baseline and parameters of severity, as well as all prognostic parameters were without significant differences (Tables 1 and 2).

2) $\mathrm{WCs} \geq 102 / 88 \mathrm{~cm}$ were more frequently recorded in males and were associated with higher rates of hypertension $(p<0.05)$. Other baseline, as well as all severity and prognostic parameters were without significant differences (Tables 1 and 2).

3) WHRs $\geq 0.90 / 0.85$ were more frequently found in males and were associated with higher rates of dyslipidemia and significantly stenosed proximal/middle coronary artery segments $(\mathrm{p}<0.05)$. Other baseline and parameters of severity, as well as all prognostic parameters were without significant differences (Tables 3 and 4).

4) WHtRs $\geq 63 / 58$ were more frequently found in females and were associated with the highest rates of arterial hypertension, heart failure and total in-hospital complications $(\mathrm{p}<0.05)$. Other baseline and parameters of severity, as well as all prognostic parameters were without significant differences (Tables 3 and 4).

5) $\mathrm{BMI}<25.0 \mathrm{~kg} / \mathrm{m}^{2}$ increased (OR 2.00, CI [1.09-3.68], $\mathrm{p}=0.026)$ and BMI $25.0-29.9 \mathrm{~kg} / \mathrm{m}^{2}$ re- duced the risk of dyspnea (OR 0.52, CI [0.30-0.91], $\mathrm{p}=0.022)$; WHR $\geq 0.90 / 0.85$ adjusted for gender increased the risk of significant proximal/middle coronary segment stenosis (OR 3.34, CI [1.13-9.86], $\mathrm{p}=0.029$ ), while $\mathrm{WHtR} \geq 63 / 58$ adjusted for hyperglycemia increased the risk of heart failure (OR 2.05, CI [1.13-3.71] $\mathrm{p}=0.017)$ and total in-hospital complications (OR 1.94, CI [1.13-3.33] p=0.017). The number of significantly stenosed coronary arteries, adjusted for LVEF and distal coronary segment stenosis increased the risk of total MACE (OR 1.79, CI [1.17-2.77], $\mathrm{p}=0.008$ ).

\section{Discussion}

The main objective of this study was to investigate the controversial influence of anthropometric parameters on clinical severity and prognosis of acute STE$\mathrm{MI}$, as well as their unknown influence on the involvement of coronary segments with significant stenosis and SLD.

We found the measures of central obesity (WHR and $\mathrm{WH}$ tR) to be superior to BMI in predicting clinical severity (significant proximal/middle coronary segment stenosis, heart failure and total in-hospital complications vs. dyspnea), while WC had no influence on it. Also, anthropometric parameters had no influence on prognosis. Finally, the number of significantly ste- 
Table 2. Severity and prognosis of acute ST-elevation myocardial infarction according to $B M I\left(\mathrm{~kg} / \mathrm{m}^{2}\right)$ and $W C(\mathrm{~cm})$

\begin{tabular}{|c|c|c|c|c|c|c|c|c|}
\hline & Parameter & $\begin{array}{c}\mathrm{BMI}<25.0 \\
\quad(\mathrm{n}=60)\end{array}$ & $\begin{array}{c}\text { BMI 25.0- } \\
29.9 \\
(n=118)\end{array}$ & $\begin{array}{c}\mathrm{BMI} \geq 30.0 \\
\quad(\mathrm{n}=72)\end{array}$ & $\mathrm{p}$ & $\begin{array}{c}\text { WC } \\
<102 / 88 \\
(n=101)\end{array}$ & $\begin{array}{c}\text { WC } \\
\geq 102 / 88 \\
(n=149)\end{array}$ & $\mathrm{p}$ \\
\hline \multirow{3}{*}{$\begin{array}{l}\text { Clinical } \\
\text { presenta- } \\
\text { tion }\end{array}$} & Angina pectoris, $\mathrm{n}(\%)$ & $60(100)$ & $114(96.6)$ & $71(98.6)$ & 0.283 & $99(98)$ & $146(98)$ & 0.985 \\
\hline & Dyspnea, n (\%) & $25(41.7)$ & $27(22.9)$ & $23(31.9)$ & 0.032 & $27(26.7)$ & $48(32.2)$ & 0.353 \\
\hline & Hospitalization (days) & $9(2-31)$ & $8.5(2-21)$ & $9(6-32)$ & 0.028 & $8(2-30)$ & $9(3-32)$ & 0.126 \\
\hline \multirow{10}{*}{$\begin{array}{l}\text { In-hospi- } \\
\text { tal com- } \\
\text { plications }\end{array}$} & Arrhythmias, n (\%) & $11(18.3)$ & $20(17)$ & $12(16.7)$ & 0.964 & $14(13.9)$ & $29(19.5)$ & 0.250 \\
\hline & $\begin{array}{l}\text { Conduction abnorm., } \\
\mathrm{n}(\%)\end{array}$ & $6(10)$ & $6(5.1)$ & $4(5.6)$ & 0.422 & $4(4)$ & $12(8.1)$ & 0.194 \\
\hline & Heart failure, n (\%) & $17(28.3)$ & $25(21.2)$ & $22(30.6)$ & 0.306 & $20(19.8)$ & $44(29.5)$ & 0.084 \\
\hline & Cardiogenic shock, n (\%) & $6(10)$ & $8(6.8)$ & $4(5.6)$ & 0.598 & $7(6.9)$ & $11(7.4)$ & 0.892 \\
\hline & Cardiac arrest, $\mathrm{n}(\%)$ & $9(15)$ & $16(13.6)$ & $11(15.3)$ & 0.937 & $12(11.9)$ & $24(16.1)$ & 0.350 \\
\hline & $\begin{array}{l}\text { Mechanical ventilation, } \\
\mathrm{n}(\%)\end{array}$ & $2(3.3)$ & $5(4.2)$ & $3(4.2)$ & 0.955 & $2(2)$ & $8(5.4)$ & 0.180 \\
\hline & Reinfarction, n (\%) & $0(0)$ & $1(0.8)$ & $0(0)$ & - & $0(0)$ & $1(0.7)$ & - \\
\hline & Re-PCI, n (\%) & $1(1.7)$ & $3(2.5)$ & $0(0)$ & - & $1(1)$ & $3(2)$ & - \\
\hline & Mortality, n (\%) & $6(10)$ & $6(5.1)$ & $7(9.7)$ & 0.365 & $10(9.9)$ & $9(8.3)$ & 0.258 \\
\hline & Total, n (\%) & $27(45)$ & $45(38.1)$ & $32(44.4)$ & 0.575 & $36(35.6)$ & $68(45.6)$ & 0.116 \\
\hline \multirow{2}{*}{$\begin{array}{l}\text { Labora- } \\
\text { tory }\end{array}$} & Max. cTnT (ng/mL) & $3.7(0-10)$ & $3(0-10)$ & $2.9(0-10)$ & 0.642 & $3.5(0-10)$ & $2.8(0-10)$ & 0.246 \\
\hline & Max. CK (U/L) & $\begin{array}{c}1915.5 \\
(107-15617) \\
\end{array}$ & $\begin{array}{c}1779 \\
(25-13769) \\
\end{array}$ & $\begin{array}{c}1900 \\
(85-14094) \\
\end{array}$ & 0.952 & $\begin{array}{c}2571 \\
(70-15617)\end{array}$ & $\begin{array}{c}1701 \\
(25-14094)\end{array}$ & 0.296 \\
\hline $\mathrm{ECHO}$ & $\begin{array}{l}\text { Left ventricle ejection } \\
\text { fraction }(\%)\end{array}$ & $50(28-70)$ & $53(25-70)$ & $50(30-76)$ & 0.949 & $50(25-64)$ & $50(28-76)$ & 0.521 \\
\hline \multirow{7}{*}{$\begin{array}{l}\text { Coronary } \\
\text { angiogra- } \\
\text { phy }\end{array}$} & Stenosed CAs & $2(1-4)$ & $2(1-4)$ & $1(1-3)$ & 0.456 & $1(1-4)$ & $2(1-4)$ & 0.399 \\
\hline & $\geq 2$ stenosed CAs, n (\%) & $33(55)$ & $60(50.8)$ & $35(48.6)$ & 0.761 & $48(47.5)$ & $80(53.7)$ & 0.339 \\
\hline & Number of stents & $1(1-4)$ & $1(1-3)$ & $1(1-3)$ & 0.266 & $1(1-4)$ & $1(1-3)$ & 0.269 \\
\hline & Stents diameter $(\mathrm{mm})$ & $3(2.5-4)$ & $3.5(2.3-4)$ & $3.5(2.8-4)$ & 0.000 & $3(2.8-4)$ & $3.5(2.3-4)$ & 0.184 \\
\hline & Stents length $(\mathrm{mm})$ & $18(8-36)$ & $20(8-38)$ & $20(8-38)$ & 0.099 & $18.5(8-38)$ & $20(8-38)$ & 0.060 \\
\hline & $\begin{array}{l}\text { Proximal/middle CA } \\
\text { segment stenosis, n (\%) }\end{array}$ & $54(90)$ & $106(90.6)$ & $66(91.7)$ & 0.944 & $88(88)$ & $138(92.6)$ & 0.217 \\
\hline & $\begin{array}{l}\text { Distal CA segment } \\
\text { stenosis, } \mathrm{n}(\%)\end{array}$ & $27(45)$ & $47(40.2)$ & $23(31.9)$ & 0.289 & $38(39.2)$ & $59(39.6)$ & 0.800 \\
\hline \multirow[t]{9}{*}{ MACE } & Reinfarction, n (\%) & $1(1.9)$ & $0(0)$ & $1(1.5)$ & - & $1(1.1)$ & $1(0.7)$ & - \\
\hline & Restenosis, n (\%) & $2(3.8)$ & $4(3.8)$ & $1(1.5)$ & 0.655 & $4(4.4)$ & $3(2.2)$ & 0.349 \\
\hline & New stenosis, n (\%) & $1(1.9)$ & $3(2.8)$ & $3(4.4)$ & 0.712 & $4(4.4)$ & $3(2.1)$ & 0.349 \\
\hline & Cardiac rehosp., n (\%) & $8(15.1)$ & $18(17)$ & $11(16.2)$ & 0.954 & $13(14.3)$ & $24(17.6)$ & 0.502 \\
\hline & $\begin{array}{l}\text { Non-cardiac rehosp., } \\
\mathrm{n}(\%)\end{array}$ & $5(9.4)$ & $3(2.8)$ & $1(1.5)$ & 0.059 & $4(4.4)$ & $5(3.7)$ & 0.786 \\
\hline & CVI, n (\%) & $1(1.9)$ & $0(0)$ & $0(0)$ & - & $0(0)$ & $1(0.7)$ & - \\
\hline & Urgent CABG, n (\%) & $1(1.9)$ & $3(2.8)$ & $2(2.9)$ & 0.925 & $1(1.1)$ & $5(3.7)$ & 0.236 \\
\hline & Mortality, n (\%) & $3(5.7)$ & $1(0.9)$ & $0(0)$ & - & $2(2.2)$ & $2(1.5)$ & - \\
\hline & Total, n (\%) & $11(20.8)$ & $20(18.4)$ & $16(23.5)$ & 0.706 & $18(19.8)$ & $29(20.9)$ & 0.842 \\
\hline Other & $\begin{array}{l}\text { Sick leave duration } \\
\text { (weeks) }\end{array}$ & $12(2-52)$ & $12(1-28)$ & $14(2-48)$ & 0.401 & $12(1-48)$ & $12(3-52)$ & 0.093 \\
\hline
\end{tabular}

$\mathrm{BMI}=$ body mass index; $\mathrm{CABG}=$ coronary artery bypass graft $\mathrm{CA}=$ coronary artery $\mathrm{CK}=$ creatinine phosphokinase; $\mathrm{cTnT}=\mathrm{cardiac}$ troponin $\mathrm{T} ; \mathrm{CVI}=$ cerebrovascular insult $\mathrm{ECHO}=$ echocardiography; $\mathrm{MACE}=$ major adverse cardiovascular events; $\mathrm{PCI}=$ percutaneous coronary intervention; $\mathrm{WC}=$ waist circumference 
Table 3. Baseline characteristics of patients according to WHtR and WHR

\begin{tabular}{|l|c|c|c|c|c|c|c|}
\hline \multirow{2}{*}{ Parameter } & $\begin{array}{c}\text { WHtR } \\
<53 / 49 \\
(\mathrm{n}=42)\end{array}$ & $\begin{array}{c}\text { WHtR } \\
53 / 49- \\
62 / 57 \\
(\mathrm{n}=127)\end{array}$ & $\begin{array}{c}\text { WHtR } \geq \\
63 / 58 \\
(\mathrm{n}=81)\end{array}$ & $\mathrm{p}$ & $\begin{array}{c}\text { WHR } \\
<0.90 / 0.85 \\
(\mathrm{n}=28)\end{array}$ & $\begin{array}{c}\text { WHR } \\
\geq 0.90 / 0.85 \\
(\mathrm{n}=222)\end{array}$ & $\mathrm{p}$ \\
\hline Men, n (\%) & $36(85.7)$ & $105(82.7)$ & $36(44.4)$ & $<\mathbf{0 . 0 0 0 1}$ & $8(28.6)$ & $169(76.1)$ & $\mathbf{0 . 0 0 0}$ \\
Women, n (\%) & $6(14.3)$ & $22(17.3)$ & $45(55.6)$ & $<\mathbf{0 . 0 0 0 1}$ & $20(71.4)$ & $53(23.9)$ & $\mathbf{0 . 0 0 0}$ \\
Age (years) & $60(42-86)$ & $60(25-92)$ & $64(39-85)$ & 0.162 & $70(39-88)$ & $61(25-92)$ & $\mathbf{0 . 0 2 1}$ \\
Arterial hypertension, n (\%) & $24(57.1)$ & $84(66.1)$ & $73(90.1)$ & $<\mathbf{0 . 0 0 0 1}$ & $21(75)$ & $160(72.1)$ & 0.744 \\
Dyslipidemia, n (\%) & $33(78.6)$ & $97(76.4)$ & $60(74.1)$ & 0.849 & $17(60.7)$ & $173(77.9)$ & $\mathbf{0 . 0 4 4}$ \\
Hyperglycemia, n (\%) & $8(19.0)$ & $29(22.8)$ & $24(29.6)$ & 0.364 & $6(21.4)$ & $55(24.8)$ & 0.698 \\
Smoking, n (\%) & $27(64.3)$ & $62(48.8)$ & $40(49.4)$ & 0.196 & $11(39.3)$ & $118(53.2)$ & 0.166 \\
Family history, n (\%) & $19(45.2)$ & $52(40.9)$ & $36(44.4)$ & 0.831 & $12(42.9)$ & $95(42.8)$ & 0.995 \\
Previous MI, n (\%) & $5(11.9)$ & $11(8.7)$ & $6(7.4)$ & 0.704 & $3(10.7)$ & $19(8.6)$ & 0.704 \\
Previous PCI, n (\%) & $6(14.3)$ & $13(10.2)$ & $4(4.9)$ & 0.199 & $2(7.1)$ & $21(9.5)$ & 0.689 \\
Previous CABG, n (\%) & $0(0)$ & $1(0.8)$ & $0(0)$ & - & $0(0)$ & $1(0.5)$ & - \\
\hline
\end{tabular}

$\mathrm{CABG}=$ coronary artery bypass graft $\mathrm{MI}=$ myocardial infarction; $\mathrm{PCI}=$ percutaneous coronary intervention; WHR = waist-to-hip ratio; $\mathrm{WH}$ tR = waist-to-height ratio

nosed coronary arteries increased the risk of total MACE, which is consistent with literature data ${ }^{12}$.

Several studies have reported a paradoxical clinical effect of elevated BMI on improved survival after primary PCI in patients with acute STEMI, i.e. the overall 'obesity paradox'. Overweight and obese patients had wider stents, normal LVEF, lower CK levels, inhospital and overall mortalities, as well as lower rates of MACE during 12-month follow up ${ }^{13-16}$.

According to other authors, obese patients with acute STEMI had similar PCI characteristics and MACE as normal weights and overweights ${ }^{17}$. Iakobishvili et al. and $\mathrm{Li}$ et al. found no significant differences in infarct size, and in 3-month and 1-year outcomes among the BMI categories with acute STEMI and primary $\mathrm{PCI}^{18,19}$.

The 'obesity paradox' could explain why we found no significant differences among normal weight, overweight and overall obese patients in prognosis, as well as that of the severity parameters, normal weight increased and overweight reduced the risk of dyspnea.

The presence of increased WC is associated with greater myocardial necrosis in patients with acute $\mathrm{MI}^{20}$. However, it has been reported that increased WC has a protective role for the presence of significant angiographic CAD, i.e. central 'obesity paradox ${ }^{21}$. Subcutaneous fat component is probably mainly re- sponsible for the paradoxical protective effect of central obesity, whereas visceral fat may have an opposing effect and increase the risk of significant angiographic CAD. WC does not add prognostic information for predicting six-month mortality or myocardial reinfarction in patients with acute $\mathrm{MI}^{22}$. In this study, we found no significant differences in any of the parameters of severity and prognosis between patients with normal and increased WC.

The presence of increased WHR is associated with significant coronary stenosis, but not with the number of significantly stenosed coronary arteries ${ }^{23}$. In acute STEMI, patients with increased WHR more frequently have heart failure and WHR $\geq 0.90 / 0.85$ is an independent predictor of six-month mortality ${ }^{24}$. In our study, where a small number of patients with normal WHR was found as expected, the presence of increased WHR was associated with significant stenosis of proximal/middle coronary segments, but without significant differences in other severity and in all prognostic parameters.

Of the anthropometric parameters, WHtR yielded the highest positive correlation with $\mathrm{CAD}^{25}$. This was the first study on the effect of WHtR on clinical severity and prognosis of acute STEMI. We found that WHtR $\geq 63 / 58$ increased the risk of heart failure and total in-hospital complications. Considering the small 
Table 4. Severity and prognosis of acute ST-elevation myocardial infarction according to $W H t R\left(\mathrm{~kg} / \mathrm{m}^{2}\right)$ and $W H R$

\begin{tabular}{|c|c|c|c|c|c|c|c|c|}
\hline & Parameter & $\begin{array}{l}\text { WHtR } \\
<53 / 49 \\
(n=42)\end{array}$ & $\begin{array}{c}\text { WHtR } \\
53 / 49-62 / 57 \\
(n=127)\end{array}$ & $\begin{array}{l}\text { WHtR } \\
\geq 63 / 58 \\
(n=81)\end{array}$ & $\mathrm{p}$ & $\begin{array}{c}\text { WHR } \\
<0.90 / 0.85 \\
(n=28)\end{array}$ & $\begin{array}{c}\text { WHR } \\
\geq 0.90 / 0.85 \\
(n=222)\end{array}$ & $\mathrm{p}$ \\
\hline \multirow{3}{*}{$\begin{array}{l}\text { Clinical } \\
\text { presenta- } \\
\text { tion }\end{array}$} & Angina pectoris, $\mathrm{n}(\%)$ & $41(97.6)$ & $124(97.6)$ & $80(98.8)$ & 0.836 & $27(96.4)$ & $218(98.2)$ & 0.529 \\
\hline & Dyspnea, n (\%) & $15(35.7)$ & $36(28.3)$ & $24(29.6)$ & 0.662 & $10(35.7)$ & $65(29.3)$ & 0.484 \\
\hline & Hospitalization (days) & $8(1-20)$ & $9(1-32)$ & $9(3-30)$ & 0.366 & $9(5-25)$ & $9(2-32)$ & 0.193 \\
\hline \multirow{10}{*}{$\begin{array}{l}\text { In-hospi- } \\
\text { tal com- } \\
\text { plications }\end{array}$} & Arrhythmias, n (\%) & $5(11.9)$ & $23(18.1)$ & $15(18.5)$ & 0.607 & $2(7.1)$ & $41(18.5)$ & 0.135 \\
\hline & $\begin{array}{l}\text { Conduction abnorm., } \\
\mathrm{n}(\%)\end{array}$ & $2(4.8)$ & $10(7.9)$ & $4(4.9)$ & 0.626 & $3(10.7)$ & $13(5.9)$ & 0.322 \\
\hline & Heart failure, n (\%) & $9(21.4)$ & $26(20.5)$ & $29(35.8)$ & 0.038 & $9(32.1)$ & $55(24.8)$ & 0.400 \\
\hline & Cardiogenic shock, n (\%) & $4(9.5)$ & $7(5.5)$ & $7(8.6)$ & 0.567 & $1(3.6)$ & $17(7.7)$ & 0.431 \\
\hline & Cardiac arrest, n (\%) & $5(11.9)$ & $17(13.4)$ & $14(17.3)$ & 0.649 & $2(7.1)$ & $34(15.3)$ & 0.246 \\
\hline & Mechanical vent., n (\%) & $1(2.4)$ & $4(3.1)$ & $5(6.2)$ & 0.467 & $0(0)$ & $10(4.5)$ & 0.252 \\
\hline & Reinfarction, n (\%) & $0(0)$ & $0(0)$ & $1(1.2)$ & - & $0(0)$ & $1(0.5)$ & - \\
\hline & Re-PCI, n (\%) & $0(0)$ & $4(3.1)$ & $0(0)$ & - & $0(0)$ & $4(1.8)$ & - \\
\hline & Mortality, n (\%) & $5(11.9)$ & $9(7.1)$ & $5(6.2)$ & 0.499 & $2(7.1)$ & $17(7.7)$ & 0.923 \\
\hline & Total, n (\%) & $12(28.6)$ & $49(38.6)$ & $43(53.1)$ & 0.020 & $10(35.7)$ & $94(42.3)$ & 0.503 \\
\hline \multirow{2}{*}{$\begin{array}{l}\text { Labora- } \\
\text { tory }\end{array}$} & Max. cTnT (ng/mL) & $3.7(0.1-10)$ & $3.7(0-10)$ & $2.6(0-10)$ & 0.061 & $2.1(0-10)$ & $3.2(0-10)$ & 0.071 \\
\hline & Max. CK(U/L) & $\begin{array}{c}2652 \\
(107-15617) \\
\end{array}$ & $\begin{array}{c}1983 \\
(25-13331) \\
\end{array}$ & $\begin{array}{c}1420 \\
(85-14094) \\
\end{array}$ & 0.118 & $\begin{array}{c}1324 \\
(85-11425)\end{array}$ & $\begin{array}{c}1926 \\
(25-15617) \\
\end{array}$ & 0.122 \\
\hline $\mathrm{ECHO}$ & $\begin{array}{l}\text { Left ventricle ejection } \\
\text { fraction }(\%)\end{array}$ & $50(25-65)$ & $50(28-76)$ & $55(30-68)$ & 0.691 & $50(30-70)$ & $50(25-76)$ & 0.944 \\
\hline \multirow{7}{*}{$\begin{array}{l}\text { Coronary } \\
\text { angiogra- } \\
\text { phy }\end{array}$} & Stenosed CAs & $1.5(1-3)$ & $1(1-4)$ & $2(1-4)$ & 0.740 & $1.5(1-4)$ & $2(1-4)$ & 0.750 \\
\hline & $\geq 2$ stenosed CAs, n (\%) & $21(50.0)$ & $62(48.8)$ & $45(55.6)$ & 0.629 & $14(50)$ & $114(51.4)$ & 0.893 \\
\hline & Number of stents & $1(1-3)$ & $1(1-4)$ & $1(1-3)$ & 0.432 & $1(1-3)$ & $1(1-4)$ & 0.119 \\
\hline & Stents diameter $(\mathrm{mm})$ & $3(2.8-4.0)$ & $\begin{array}{c}3.5 \\
(2.5-4.0) \\
\end{array}$ & $\begin{array}{c}3.5 \\
(2.3-4.0) \\
\end{array}$ & 0.062 & $3(2.8-4)$ & $3.5(2.3-4)$ & 0.515 \\
\hline & Stents length $(\mathrm{mm})$ & $20(8-36)$ & $20(12-38)$ & $20(12-36)$ & 0.291 & $20(8-36)$ & $20(8-38)$ & 0.905 \\
\hline & $\begin{array}{l}\text { Proximal/middle CA } \\
\text { segment stenosis, n (\%) }\end{array}$ & $37(88.1)$ & $113(89.7)$ & $76(93.8)$ & 0.487 & $21(75)$ & $205(92.8)$ & 0.002 \\
\hline & $\begin{array}{l}\text { Distal CA segment } \\
\text { stenosis, } \mathrm{n}(\%)\end{array}$ & $15(35.7)$ & $51(40.5)$ & $31(38.3)$ & 0.850 & $15(53.6)$ & $82(37.1)$ & 0.092 \\
\hline \multirow[t]{9}{*}{ MACE } & Reinfarction, n (\%) & $0(0)$ & $1(0.9)$ & $1(1.3)$ & - & $0(0)$ & $2(1)$ & - \\
\hline & Restenosis, n (\%) & $2(5.4)$ & $5(4.3)$ & $0(0)$ & - & $1(3.7)$ & $6(3)$ & - \\
\hline & New stenosis, n (\%) & $2(5.4)$ & $3(2.6)$ & $2(2.6)$ & 0.659 & $2(7.4)$ & $5(2.5)$ & - \\
\hline & Cardiac rehosp., n (\%) & $3(8.1)$ & $22(18.8)$ & $12(15.8)$ & 0.303 & $2(7.4)$ & $35(17.5)$ & 0.183 \\
\hline & $\begin{array}{l}\text { Non-cardiac rehosp., } \\
\mathrm{n}(\%)\end{array}$ & $1(2.7)$ & $6(5.1)$ & $2(2.6)$ & 0.626 & $1(3.7)$ & $8(4)$ & 0.941 \\
\hline & CVI, n (\%) & $0(0)$ & $1(0.9)$ & $0(0)$ & - & $0(0)$ & $1(0.5)$ & - \\
\hline & Urgent CABG, n (\%) & $0(0)$ & $4(3.4)$ & $2(2.6)$ & - & $0(0)$ & $6(3)$ & - \\
\hline & Mortality, n (\%) & $0(0)$ & $3(2.6)$ & $1(1.3)$ & - & $0(0)$ & $4(2)$ & - \\
\hline & Total, n (\%) & $5(13.5)$ & $28(23.9)$ & $14(18.4)$ & 0.340 & $7(25.9)$ & $40(19.7)$ & 0.451 \\
\hline Other & $\begin{array}{l}\text { Sick leave duration } \\
\text { (weeks) }\end{array}$ & $16(2-24)$ & $12(1-52)$ & $12(3-40)$ & 0.118 & $12(10-26)$ & $12(1-52)$ & 0.656 \\
\hline
\end{tabular}

$\mathrm{CABG}=$ coronary artery bypass graft $\mathrm{CA}=$ coronary artery $\mathrm{CK}=$ creatinine phosphokinase; $\mathrm{cTnT}=$ cardiac troponin $\mathrm{T}$; CVI = cerebrovascular insult; $\mathrm{ECHO}=$ echocardiography; $\mathrm{MACE}$ = major adverse cardiovascular events; $\mathrm{PCI}$ = percutaneous coronary intervention; $\mathrm{WHR}=$ waist-to-hip ratio; $\mathrm{WHtR}=$ waist-to-height ratio 
number of patients with normal WHR and WHtR values as the main limitation of this study, investigation with a greater number of patients should be performed to confirm these results.

\section{Conclusion}

In conclusion, WHR and WHtR are better anthropometric parameters than BMI in predicting acute STEMI severity, while WC has no influence on it. Anthropometry has no influence on prognosis.

\section{References}

1. World Health Organization. Obesity and overweight. Fact sheet $\mathrm{N}^{\circ} 311$, updated March 2013. Available at: http://www. who.int/mediacentre/factsheets/fs311/en/

2. Berghöfer A, Pischon T, Reinhold T, Apovian CM, Sharma AM, Willich SN. Obesity prevalence from a European perspective: a systematic review. BMC Public Health. 2008;8:200. http://dx.doi.org/10.1186/1471-2458-8-200.

3. Artham SM, Lavie CJ, Milani RV, Ventura HO. Obesity and hypertension, heart failure, and coronary heart disease-risk factor, paradox, and recommendations for weight loss. Ochsner J. 2009;9(3):124-32.

4. Coutinho T, Goel K, Corrêa de Sá D, Kragelund C, Kanaya $\mathrm{AM}$, Zeller M, et al. Central obesity and survival in subjects with coronary artery disease: a systematic review of the literature and collaborative analysis with individual subject data. J Am Coll Cardiol. 2011;57(19):1877-86. http://dx.doi.org/ 10.1016/j.jacc.2010.11.058.

5. Browning LM, Hsieh SD, Ashwell M. A systematic review of waist-to-height ratio as a screening tool for the prediction of cardiovascular disease and diabetes: 0.5 could be a suitable global boundary value. Nutr Res Rev. 2010;23(2):247-69. http://dx.doi.org/10.1017/S0954422410000144.

6. Lee CD, Jacobs DR Jr, Schreiner PJ, Iribarren C, Hankinson A. Abdominal obesity and coronary artery calcification in young adults: the Coronary Artery Risk Development in Young Adults (CARDIA) Study. Am J Clin Nutr. 2007;86(1):48-54.

7. Van de Werf F, Bax J, Betriu A, Blomstrom-Lundquist C, Crea F, Falk V, et al.; ESC Committee for Practice Guidelines (CPG). Management of acute myocardial infarction in patients presenting with persistent ST-segment elevation: the Task Force on the Management of ST-Segment Elevation Acute Myocardial Infarction of the European Society of Cardiology. Eur Heart J. 2008;29(23):2909-45. http://dx.doi.org/10.1093/ eurheartj/ehn416.

8. Task Force on Myocardial Revascularization of the European Society of Cardiology (ESC) and the European Association for Cardio-Thoracic Surgery (EACTS); European Association for Percutaneous Cardiovascular Interventions (EAPCI), Wijns
W, Kolh P, Danchin N, Di Mario C, Falk V, et al. Guidelines on myocardial revascularization. Eur Heart J. 2010;31(20): 2501-55. http://dx.doi.org/10.1093/eurheartj/ehq277.

9. Schneider HJ, Glaesmer H, Klotsche J, Böhler S, Lehnert H, Zeiher AM, et al.; DETECT Study Group. Accuracy of anthropometric indicators of obesity to predict cardiovascular risk. J Clin Endocrinol Metab. 2007;92(2):589-94. http://dx. doi.org/10.1210/jc.2006-0254.

10. Cheitlin MD, Armstrong WF, Aurigemma GP, Beller GA, Bierman FZ, Davis JL, et al.; American College of Cardiology; American Heart Association; American Society of Echocardiography. ACC/AHA/ASE 2003 guideline update for the clinical application of echocardiography: summary article: a report of the American College of Cardiology/American Heart Association Task Force on Practice Guidelines (ACC/AHA/ ASE Committee to Update the 1997 Guidelines for the Clinical Application of Echocardiography). Circulation. 2003;108 (9):1146-62. http://dx.doi.org/ 10.1161/01.CIR.0000073597. 57414.A9.

11. Austen WG, Edwards JE, Frye RL, Gensini GG, Gott VL, Griffith LS, et al. A reporting system on patients evaluated for coronary artery disease. Report of the Ad Hoc Committee for Grading of Coronary Artery Disease, Council on Cardiovascular Surgery, American Heart Association. Circulation. 1975;51 (4 Suppl):5-40.

12. Sorajja P, Gersh BJ, Cox DA, McLaughlin MG, Zimetbaum P, Costantini $\mathrm{C}$, et al. Impact of multivessel disease on reperfusion success and clinical outcomes in patients undergoing primary percutaneous coronary intervention for acute myocardial infarction. Eur Heart J. 2007;28(14):1709-16. http://dx.doi. org/10.1093/eurheartj/ehm184.

13. Kosuge M, Kimura K, Kojima S, Sakamoto T, Ishihara M, Asada Y, et al.; Japanese Acute Coronary Syndrome Study (JACSS) Investigators. Impact of body mass index on in-hospital outcomes after percutaneous coronary intervention for ST segment elevation acute myocardial infarction. Circ J. 2008; 72(4):521-5. http://doi.org/10.1253/circj.72.521.

14. Kang WY, Jeong MH, Ahn YK, Kim JH, Chae SC, Kim YJ, et al.; Korea Acute Myocardial Infarction Registry Investigators. Obesity paradox in Korean patients undergoing primary percutaneous coronary intervention in ST-segment elevation myocardial infarction. J Cardiol. 2010;55(1):84-91. http://dx.doi. org/10.1016/j.jjcc.2009.10.004.

15. Foley DP, Melkert R, Serruys PW. Influence of coronary vessel size on renarrowing process and late angiographic outcome after successful balloon angioplasty. Circulation.1994;90(3):123951. http://dx.doi.org/10.1161/01.CIR.90.3.1239.

16. Lancefield T, Clark DJ, Andrianopoulos N, Brennan AL, Reid CM, Johns J, et al.; MIG (Melbourne Interventional Group) Registry. Is there an obesity paradox after percutaneous coronary intervention in the contemporary era? An analysis from a multicenter Australian registry. JACC Cardiovasc Interv. 2010; 3(6):660-8. http://dx.doi.org/10.1016/j.jcin.2010.03.018.

17. Sun Y, Jiang D, Zhang B, Yu H, Gao Y, Li Y, et al. Impact of obesity on the outcome of Chinese patients with ST-segment 
myocardial infarction undergoing urgent percutaneous coronary intervention. Acta Cardiol. 2012;67(5):541-8. http://dx. doi.org/10.2143/AC.67.5.2174128.

18. Iakobishvili Z, Danicek V, Porter A, Assali AR, Battler A, Hasdai D. Is increased body mass index associated with a cardioprotective effect after ST-segment-elevation myocardial infarction? Acute Card Care. 2006;8(2):95-8. http://dx.doi. org/10.1080/17482940600768673.

19. Li YZ, Sun YJ, Zhang B, Jiang DM, Gao Y, Yu HJ, et al. The impact of body mass index on the presentation, treatment and clinical outcomes of patients with ST-segment elevated myocardial infarction. Zhonghua Liu Xing Bing Xue Za Zhi. 2012;33(12):1288-92. (in Chinese). http://dx.doi.org/10.3760/ cma.j.issn.0254-6450.2012.12.021.

20. Iglesias Bolaños P, Olivar Roldán J, Peñalver Talavera D, Díaz Guardiola P, Vega Piñero B, Monereo Megías S. Effect of abdominal obesity on size of myocardial infarction. Endocrinol Nutr. 2009;56(1):4-8. (in Spanish). http://dx.doi.org/10.1016/ S1575-0922(09)70187-1.

21. Bechlioulis A, Vakalis K, Naka KK, Bourantas CV, Papamichael ND, Kotsia A, et al. Paradoxical protective effect of central obesity in patients with suspected stable coronary artery disease. Obesity (Silver Spring).2013;21(3):E314-21. http:// dx.doi.org/10.1002/oby.20074.

22. Martins A, Ribeiro S, Gonçalves P, Correia A. Role of central obesity in risk stratification after an acute coronary event: does central obesity add prognostic value to the Global Registry of Acute Coronary Events (GRACE) risk score in patients with acute coronary syndrome? Rev Port Cardiol. 2013;32(10):769-76.

23. Zen V, Fuchs FD, Wainstein MV, Gonçalves SC, Biavatti K, Riedner CE, et al. Neck circumference and central obesity are independent predictors of coronary artery disease in patients undergoing coronary angiography. Am J Cardiovasc Dis. 2012; 2(4):323-30.

24. Lee SH, Park JS, Kim W, Shin DG, Kim YJ, Kim DS, et al.; Korean Acute Myocardial Infarction Registry Investigators. Impact of body mass index and waist-to-hip ratio on clinical outcomes in patients with ST-segment elevation acute myocardial infarction (from the Korean Acute Myocardial Infarction Registry). Am J Cardiol. 2008;102(8):957-65. http://dx.doi. org/10.1016/j.amjcard.2008.06.022.

25. Sabah KM, Chowdhury AW, Khan HI, Hasan AT, Haque S, Ali $\mathrm{S}$, et al. Body mass index and waist/height ratio for prediction of severity of coronary artery disease. BMC Res Notes. 2014;7:246. http://dx.doi.org/10.1186/1756-0500-7-246.

\title{
Sažetak \\ ULOGA ANTROPOMETRIJE U AKUTNOM INFARKTU MIOKARDA SA ST-ELEVACIJOM LIJEČENOM PRIMARNOM PERKUTANOM KORONARNOM INTERVENCIJOM
}

\author{
M. Mornar Jelavić, Z. Babić, H. Pintarić i M. Mišigoj-Duraković
}

Cilj ove studije bio je istražiti proturječni utjecaj antropometrije na kliničku težinu i prognozu akutnoga infarkta miokarda sa ST-elevacijom (STEMI). Prospektivno smo analizirali 250 bolesnika s akutnim STEMI liječenih primarnom perkutanom koronarnom intervencijom (rujan 2011. - rujan 2012.). Oni su grupirani prema antropometrijskim parametrima: indeks tjelesne mase (BMI) $\left(<25,0 ; 25,0-29,9 ; \geq 30,0 \mathrm{~kg} / \mathrm{m}^{2}\right)$, opseg struka $(\mathrm{WC})(<102 / 88, \geq 102 / 88 \mathrm{~cm})$, omjer struk/bokovi (WHR) $(<0,90 / 0,85, \geq 0,90 / 0,85)$ i struk/tjelesna visina (WHtR) $(<53 / 49,53 / 49-62 / 57, \geq 63 / 58)$. Skupine su analizirane prema bazičnim te parametrima težine (klinički, laboratorijski, ehokardiografski, koronarografski, bolničke komplikacije) i prognoze (glavni neželjeni kardiovaskularni događaji (MACE) i duljina bolovanja (SLD) tijekom 12 mjeseci praćenja). Bolesnici s BMI $<25,0 \mathrm{~kg} / \mathrm{m}^{2}$ imali su najveću učestalost dispneje, a oni s BMI $\geq 30,0 \mathrm{~kg} / \mathrm{m}^{2}$ najdulju hospitalizaciju i najšire stentove; bolesnici s WHR $\geq 0,90 / 0,85$ učestalije su imali značajno stenozirane proksimalne/srednje koronarne segmente, dok su oni s WHtR $\geq 63 / 58$ imali najveću učestalost srčanog zatajivanja i ukupnih bolničkih komplikacija $(\mathrm{p}<0,05)$. BMI $<25,0 \mathrm{~kg} / \mathrm{m}^{2}$ povećava (odds ratio (OR) 2,00, confidence interval (CI) $[1,09-3,68], \mathrm{p}=0,026$ ), a BMI $25,0-29,9 \mathrm{~kg} / \mathrm{m}^{2}$ smanjuje (OR 0,52, CI $[0,30-0,91], \mathrm{p}=0,022)$ rizik dispneje; WHR $\geq 0.90 / 0.85$ povećava rizik značajne stenoze proksimalnih/srednjih koronarnih segmenata (OR 3,34, CI [1,13-9,86], p=0,029), a WHtR $\geq 63 / 58$ srčanog zatajenja (OR 2,05, CI [1,13-3,71], $\mathrm{p}=0,017)$ i ukupnih bolničkih komplikacija (OR 1,94, CI $[1,13-3,33], \mathrm{p}=0,017)(\mathrm{p}<0,05)$. Zaključno, WHR i WHtR su bolji antropometrijski parametri od BMI u predviđanju težine akutnog STEMI, dok WC nema utjecaja. Antropometrija nema utjecaja na prognozu.

Ključne riječi: Antropometrija; Pretilost; Srčani infarkt; Perkutana koronarna intervencija; Bolovanje 